\title{
Special considerations for placement of an inflatable penile prosthesis for the patient with Peyronie's disease: techniques and patient preference
}

\author{
This article was published in the following Dove Press journal: \\ Medical Devices: Evidence and Research \\ 27 July 2015 \\ Number of times this article has been viewed
}

\section{Matthew D Lyons \\ Culley C Carson III \\ Robert M Coward}

Department of Urology, University of North Carolina, Chapel Hill, NC, USA
Correspondence: Matthew D Lyons Department of Urology, University of North Carolina Hospitals, I0I Manning Drive, Chapel Hill, NC 275I4, USA

Emailmlyons@unch.unc.edu

\begin{abstract}
Placement of an inflatable penile prosthesis (IPP) is the mainstay of surgical treatment for patients with Peyronie's disease (PD) and concomitant medication-refractory erectile dysfunction. Special considerations and adjunctive surgical techniques during the IPP procedure are often required for patients with PD to improve residual penile curvature, as well as postoperative penile length. The surgical outcomes and various adjunctive techniques are not significantly different from one another, and selection of the appropriate technique must be tailored to patient-specific factors including the extent of the deformity, the degree of penile shortening, and preoperative patient expectations. The aims of this review were to assess the current literature on published outcomes and surgical techniques involving IPP placement in the treatment of PD. Patient satisfaction and preferences are reported, along with the description and patient selection for surgical techniques that include manual penile modeling, management of refractory curvature with concurrent plication, and correction of severe residual curvature and penile shortening with tunica release and plaque incision and grafting. A thorough description of the available techniques and their associated outcomes may help guide surgeons to the most appropriate choice for their patients.
\end{abstract}

Keywords: Peyronie's disease, outcomes, inflatable penile prosthesis, patient expectation, patient satisfaction

\section{Introduction}

Peyronie's disease (PD) is a connective tissue disorder affecting the tunica albuginea of the corpora cavernosa that results in plaque formation, associated curvature deformity with erections and, often, erectile dysfunction (ED). While medical management strategies exist, those who experience worsening or refractory disease require surgical management. The goals of surgery are to straighten the curvature deformity, preserve or restore erectile function, and preserve penile length and girth, if possible. ${ }^{1,2}$ For those patients who suffer from combined PD as well as medication-refractory ED, prosthesis surgery is recommended as a first-line surgical therapy. ${ }^{3-6}$ Among several types of penile prostheses, the inflatable penile prosthesis (IPP) is the best penile prosthesis for patients with PD due to its ability to improve erectile straightening and penile length. The use of penile prosthesis surgery for the management of PD was first described by Scott et $\mathrm{al}^{7}$ and was later popularized by Wilson and Delk in the early 1990s. ${ }^{8}$ IPP placement addresses both the issue of curvature deformity, as well as the issue of ED in a single procedure. Tunical scarring and corporal fibrosis prevalent in PD can make 
device placement far more challenging than in those without PD. Patients with residual curvature after prosthesis placement may be suitable for several adjunctive techniques that enable the surgeon to achieve a satisfactory result.

Success rates following IPP surgery range from $84 \%-100 \%$ with possible complications including infection, penile shortening, diminished sensitivity, device operation difficulty, persistent curvature, and mechanical failure of the prosthesis. ${ }^{3,5,6,9-15}$ Patient dissatisfaction seems to be most closely related to penile shortening, residual curvature postimplantation, and partner dissatisfaction. In this review, we discuss outcomes and patient satisfaction following IPP placement for PD, as well as special surgical considerations and adjunctive techniques utilized to address persistent curvature after IPP placement.

\section{Methods}

A PubMed literature search was conducted to identify relevant, peer-reviewed original research and review articles published between July 1973 and May 2014 related to surgical outcomes and techniques utilizing IPP for the management of PD in men with concomitant ED. Search terms for this nonsystematic review included "Peyronie's disease", "outcomes", "inflatable penile prosthesis", "patient expectation", and "patient satisfaction". Search terms were searched both separately and in combination. Thirty-eight articles were selected and analyzed. Case studies and editorials were excluded, while original research manuscripts and reviews were included, and the bibliographies of articles of interest were reviewed.

\section{Technical outcomes \\ and patient satisfaction}

Prosthesis surgery, when compared to tunical shortening or tunical lengthening procedures plus or minus erectogenic therapies, allows for more rapid development of consistent and rigid erections that help contribute to a high level of satisfaction for patients and their partners. Inflatable devices have been shown to lead to higher functional satisfaction and lower rates of persistent penile curvature deformity compared with malleable devices. ${ }^{12}$ Factors contributing to patient satisfaction include the degree of postoperative pain and swelling, postoperative complications, ease of concealment, cosmetic outcome, device functionality, ease of use, and partner acceptance. ${ }^{12}$

While patient satisfaction after IPP placement in the general population is quite high, satisfaction rates have been found to be significantly lower in those with PD.
Success rates with IPP implantation range from $84 \%$ to $100 \%$ (Table 1), ${ }^{5,6,13-15}$ while success rates in the general population range from $91 \%$ to $100 \%{ }^{5,13,15}$ Higher rates of postimplant dissatisfaction in men with PD compared to the general population have been well documented. A 5-year follow-up study looking at patient-partner satisfaction following semirigid prosthesis placement reported dissatisfaction associated with altered penile dimension, reduced penile sensitivity, poor concealment, and persistent penile deviation in men who underwent semirigid prosthesis implantation. Partner dissatisfaction in this series was related to poor penile girth, sensation of unnatural intercourse, and dyspareunia. ${ }^{16}$

Results with inflatable devices have been more favorable. A study investigating outcomes after AMS 700 CX (American Medical Systems, Inc., Minnetonka, MN, USA) IPP placements reported complete penile straightening in $70 \%$ at 6-week follow-up, with penile rigidity considered optimal by all patients. However, $30 \%$ of patients were dissatisfied with penile length, and $25 \%$ of sexual partners were not completely satisfied after 23 months of follow-up. ${ }^{17}$ In another study of 114 patients undergoing prosthesis surgery, which used the validated International Index of Erectile Function questionnaire before and after surgery in addition to the Erectile Dysfunction Inventory of Treatment Satisfaction, men with PD were noted to have significantly lower rates of satisfaction compared to the general penile implant population (relative risk =4.2). These results are likely related to decreased penile dimensions in the PD group. Patients in this study all underwent implantation of a three-piece IPP. ${ }^{18}$

Device selection does not appear to significantly affect either functional outcomes or patient satisfaction between three-piece IPPs. A study comparing outcomes following placement of the AMS 700 CX (American Medical Systems, Inc.) versus the Coloplast Titan (Coloplast Corp, Minneapolis, MN, USA) IPP in PD patients reported similar outcomes between the two devices. ${ }^{19}$ Both provided permanent penile straightening and high patient satisfaction without an increased risk of revision surgery, even after modeling. ${ }^{19}$

The most common complications include infection, penile shortening, diminished sensitivity, device operation difficulty, persistent curvature, and mechanical failure of the prosthesis. A study by Levine and Dimitriou ${ }^{6}$ looking at 90 patients at a mean follow-up of 49 months noted mechanical failure in $7.7 \%$, device bending and erosion in $2.2 \%$, and device infection in $1 \%$. Local infection rates have been reported in approximately $8 \%$ of patients undergoing prosthesis implantation for PD. ${ }^{15}$ Glans paresthesias have been reported in 5\%, and preputial edema necessitating repeat circumcision in $10 \%{ }^{13}$ 


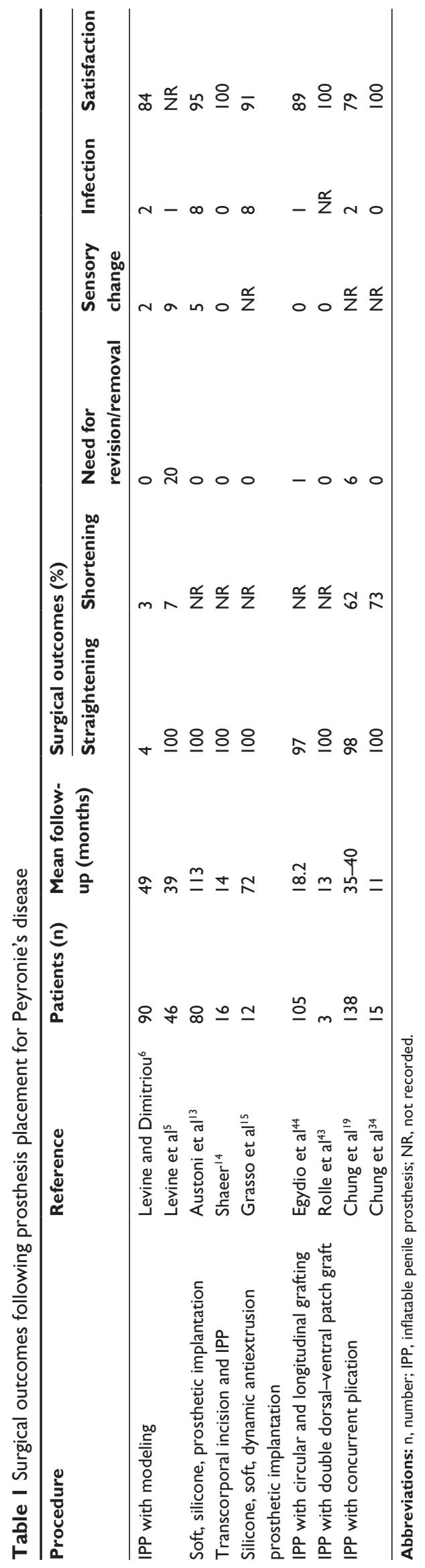


A long-term multicenter study investigating outcomes of the AMS 700 CX penile prosthesis in men with PD versus those who underwent device placement for other reasons reported higher rates of cylinder erosion in the PD group compared to the overall population ( $10.5 \%$ versus $2.3 \%$, respectively), while other morbidities were no different. Satisfaction rates were excellent in both groups, with $76 \%$ of PD patients having stated they would undergo device placement again, and approximately two-thirds of PD patients using their device twice monthly for intercourse. ${ }^{20}$

\section{Medicolegal considerations}

While overall patient satisfaction is high following prosthesis surgery, the procedure itself carries a real risk of medicolegal liability. Studies have demonstrated that the highest volume of malpractice claims occur in surgical- and/or procedural-based fields, and urology saw the eighth highest volume of claims out of 25 examined specialties. ${ }^{21}$ The average urologist can expect to be sued twice in his or her career. ${ }^{21,22}$ Patient quality of life is of particular concern in the PD and ED population, as these disease states can have a dramatic impact on patient quality of life, and the outcomes of surgical attempts to improve these issues may not meet patient expectations. The inability to fully discuss patient expectations and procedure objectives can open the door for patient dissatisfaction and subsequent legal action. A recent study reviewing legal databases for factors associated with malpractice litigation surrounding IPP implantation identified that $42 \%$ of cases (17/40) led to indemnity payment to the plaintiff with a mean settlement of US $\$ 335,000$ and a mean indemnity award of US $\$ 831,050$. Additionally, the most common breach of duty was error in surgical decision making ( $48 \%$ of cases), informed consent $(31 \%)$, and postoperative infection $(31 \%) .{ }^{23}$ These findings illustrate the importance of thorough preoperative counseling and management of patient expectations.

\section{Techniques}

Penile prosthesis placement for the treatment of PD follows similar principles as prosthesis placement in the absence of PD. The primary difference is the need for device modeling and concurrent procedures to rectify persistent curvature deformity after initial device placement. Furthermore, extensive scarring of the tunica albuginea and corporal fibrosis can pose a significant challenge to prosthesis placement in the PD patient, and in many cases this procedure requires longer corporotomies, distal counter incisions, corporal excavation and, in extreme cases, total phallic reconstruction with free flaps. ${ }^{24}$ Inflatable devices are preferred given the higher levels of functional satisfaction and lower rates of persistent curvature deformity compared with malleable devices. ${ }^{25}$ Special consideration is often required during IPP placement in PD patients due to the presence of corporal fibrosis. Several techniques have been described to navigate this challenge, including scar incision or excision, corporotomies with or without grafting, the use of cavernotomes, implant downsizing, and transcorporeal resections. ${ }^{26,27}$

Success rates for the correction of penile curvature deformity with IPP placement alone have been documented to be as low as 4\%. ${ }^{6}$ Adjunctive techniques following or during IPP placement to correct residual curvature have been described. These include: manual modeling; concurrent plication; plaque-releasing incisions; and tunical grafting procedures. Additionally, several transcorporal techniques have been developed in an attempt to simplify the release of the scar and to reduce the need for extensive modeling, plaque incision, and grafting. These include the "scratch" technique, ${ }^{28}$ and transcorporal incision. ${ }^{14}$ Guidelines and a treatment algorithm for prosthesis surgery have been described. ${ }^{4,6}$ For patients with simple a curvature of $<30^{\circ}$, manual modeling typically suffices. In the event a curvature deformity is complex, severe $\left(>30^{\circ}\right)$, or persists after manually modeling a plaque, then a releasing incision is recommended. Tunical grafting is recommended if the remaining defect is greater than $2.0 \mathrm{~cm}$ in order to prevent device herniation and circumferential contracture. ${ }^{4,29,30}$

\section{Preoperative evaluation}

Adequate preoperative evaluation is essential both in assisting the surgeon as to which technique would be most beneficial to address the needs of the patient and in providing the patient (and his partner) with the goals of surgery and the appropriate postoperative expectations. Setting proper expectations is perhaps the most important aspect of the preoperative evaluation. A thorough history and physical examination should be performed, as well as preoperative penile duplex ultrasound combined with intracavernous injection of a vasoactive agent. The use of ultrasound enables the surgeon to assess the nature and location of the Peyronie's plaque, as well as the penile vascular anatomy. Preoperative photographs of the erect penis and/or measurements of curvature should also be taken to document penile length, as well as the nature and degree of curvature. It is critical to manage patient expectations regarding postoperative penile length and appearance. This is important because many patients may have unrealistic recollections of their erect penile length prior to the onset of PD. 


\section{Manual modeling}

In the event that significant residual curvature persists following test inflation of the device, penile straightening by manually molding or "modeling" the penis should be attempted (Figures 1-4). Initially described by Wilson and Delk in 1994, modeling is an effective technique for addressing residual curvature postdevice placement. ${ }^{8}$ With the cylinders fully inflated, the shaft is bent opposite the direction of curvature to rupture the plaque and resolve the curvature. It is important that the exit tubing is clamped to prevent backflow to the pump and to compress the corporotomy sites to prevent cylinder blowout during bending. This procedure can be repeated until the curvature has been resolved. ${ }^{8}$ Penile modeling requires a high-pressure cylinder, such as the AMS 700 CX (American Medical Systems, Inc.) or Mentor Alpha-1 (Mentor Corporation, Santa Barbara, CA, USA) as studies investigating lower pressure cylinders such as the AMS 700 Ultrex cylinder (American Medical Systems, Minnetonka, MN, USA) and the Mentor Bioflex cylinder (Mentor Worldwide LLC, Santa Barbara, CA, USA) have induced aneurysmal dilatations. ${ }^{9} 10$ Modeling is the most commonly utilized technique for penile straightening in patients with mild residual curvature after device placement. ${ }^{31}$

In their initial study, Wilson and Delk ${ }^{8}$ described 138 men with PD who underwent IPP placement and subsequent modeling, and they reported straight, rigid erections in 118 men $(86 \%)$. At a mean follow-up of 32 months, $90 \%$ were using the prosthesis, and none reported penile shortening or impairment of glanular sensation. ${ }^{8}$ Complications included urethral perforation in four patients $(3 \%)$ and infection in four patients $(3 \%)$. Additional studies investigating manual modeling after the insertion of the AMS $700 \mathrm{CX}$ prostheses (American Medical Systems, Inc.) have been described with success rates ranging from $35 \%$ to $100 \% \cdot{ }^{11-14} \mathrm{Carson}^{11}$ reported that

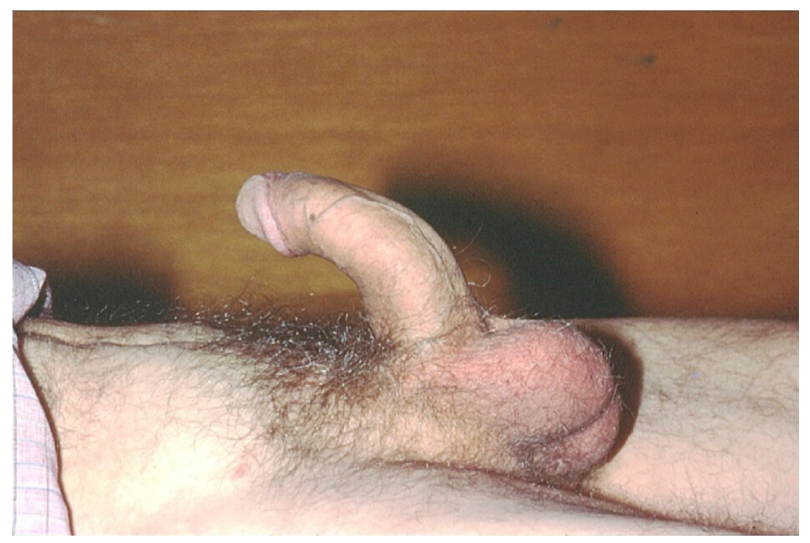

Figure I Preoperative Peyronie's disease with dorsal curvature.

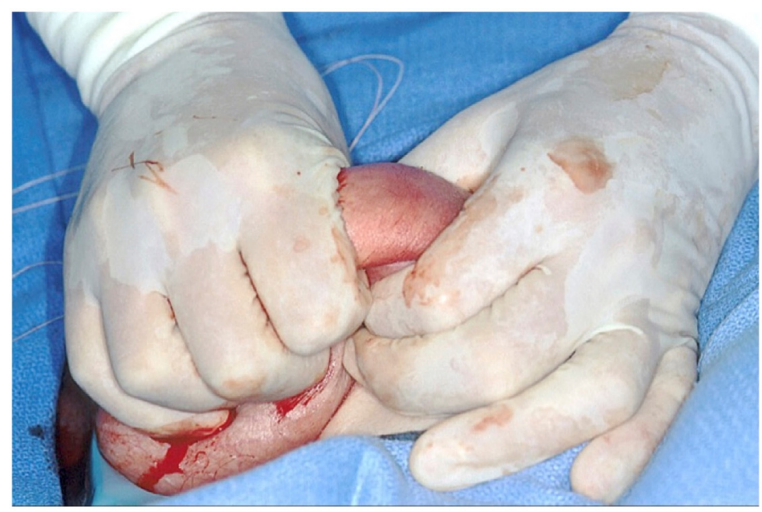

Figure 2 Intraoperative modeling postdevice placement.

28 of 30 patients who underwent the penile straightening and modeling procedure achieved complete straightening with modeling alone. Additional procedures including glanuloplasty were required in two patients, and plaque incision and graft placement was required in one patient. No patient had a postoperative infection, mechanical malfunction, or experienced urethral erosion at the 3-32-month follow-up period. ${ }^{11}$

A more recent study from 2011 by Garaffa et al ${ }^{32}$ describes successful straightening via modeling in 50 out of 62 patients (84\%) with insertion of an IPP compared with seven out of $13(54 \%)$ of those who received a malleable device. In this study, all patients' curvatures were assessed preoperatively via intracavernosal injection of prostaglandin E1. After insertion of the prostheses, residual curvature was assessed by inflating to $\sim 80 \%$ of maximal capacity. Patients with a residual curvature greater than $10^{\circ}-20^{\circ}$ underwent penile modeling. Complications included infection of the inflatable prosthesis in three patients $(2 \%)$, residual curvature in four $(3 \%)$ patients, pump failure in four patients $(3 \%)$, tubing rupture in two patients $(1.5 \%)$, damage to the

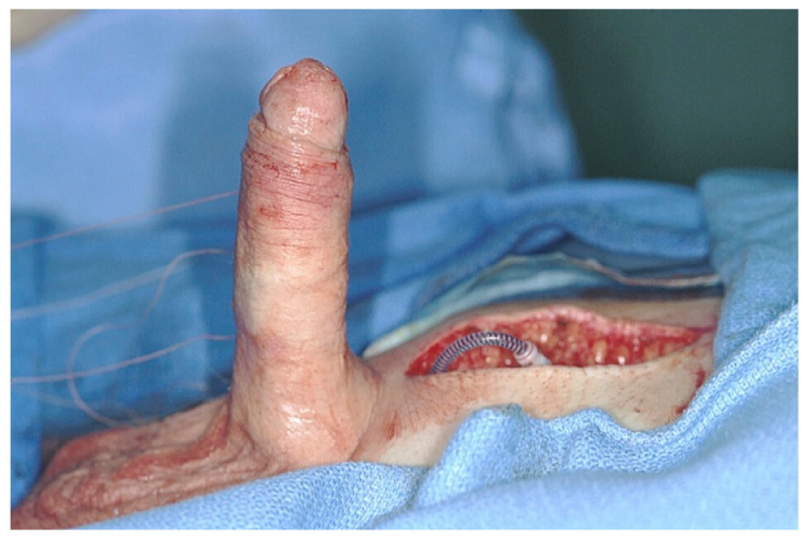

Figure 3 Penile straightening postdevice placement and modeling. 


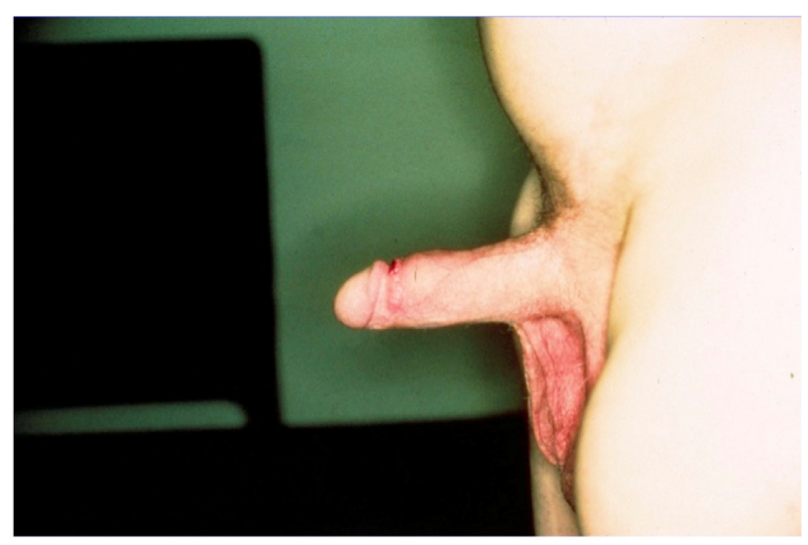

Figure 4 Activated inflatable penile prosthesis at 6 months postoperatively without residual curvature.

cylinder in one patient, soft glans in two patients (1.5\%), autoinflation in three $(2 \%)$ patients, crossover and pump malpositioning in one patient, and overall revision surgery in 18 patients $(14 \%))^{32}$

\section{Refractory curvature after manual modeling Concurrent plication}

In instances where curvature deformity is prominent or severe $\left(>30^{\circ}\right)$, synchronous plication at the time of IPP insertion has been described with good functional outcomes. This technique represents another minimally invasive method for correcting penile curvature associated with PD. Clinical scenarios in which concurrent plication have been successful include biplanar and curvature $>60^{\circ} .{ }^{33,34}$ The advantages of plication compared with plaque incision and grafting include enhanced rigidity, enhanced sensation, and fewer palpable nodules compared to those undergoing grafting procedures. $^{35}$

In a retrospective study reviewing technical and patientreported outcomes following synchronous plication during IPP placement in 18 patients, Chung et $\mathrm{al}^{36}$ report patient-perceived improvement in overall condition and penile curvature. No patients reported continued pain or required suture release at 11 months. Patients underwent plication through the standard transverse or vertical upper scrotal incision, and a series of parallel, 2-0 braided nylon sutures were placed in the tunica albuginea in an inverting, interrupted fashion. Following plication, patients underwent immediate IPP placement with the corporotomies performed well proximal to the plication sutures. The plication sutures corrected the curvature and IPP placement corrected the ED. Those authors prefer plication to modeling in cases of complex deformity. ${ }^{36}$

\section{Correction of severe curvature and penile shortening}

Additional therapeutic solutions for persistent, severe curvature include tunica release and plaque excision and grafting. These procedures are performed concurrently with IPP placement with the goal of improving penile length and reducing curvature deformity. Various different techniques of graft placement following plaque incision or excision are numerous. Devine and Horton ${ }^{37}$ utilized dermal skin grafting after plaque excision to fill the corporal defect with good success in appropriately selected patients. Lue and El-Sakka ${ }^{38}$ have described an H-shaped tunica albuginea-releasing incision on the plaque and graft placement. Modeling and concurrent plication have been shown to correct persistent curvature deformity; however, they do not address penile shortening, and plication has the potential to decrease penile length.

This has been addressed via several techniques including division of the suspensory ligament, ${ }^{39}$ plaque-releasing incision with or without grafting,,$^{24,40}$ and the use of variable geometry prostheses or implantation of a temporary intracavernous expander. ${ }^{41}$ In a study by Montorsi et al, ${ }^{17}$ 200 consecutive patients with PD who underwent placement of AMS 700 LGX (American Medical Systems, Inc.) implants and their partners were followed for a mean of 59 months and demonstrated up to $2 \mathrm{~cm}$ of length expansion. ${ }^{17}$

\section{Tunica release and plaque incision and grafting techniques}

Many authors describe achieving penile length via relaxing incisions placed in the tunica albuginea at the time of prosthesis placement. Rigaud et $\mathrm{al}^{40}$ describe fitting the corpora cavernosa with an appropriately sized nonlengthening inflatable prosthesis and lengthening the penis by performing multiple bilateral transverse incisions on the tunica albuginea, or a circular incision around the penis including the septum. A rear tip extender is then added to the prosthesis after lengthening to ensure a proper fit; the corporal defect is either left uncovered or covered with a polytetrafluoroethylene patch. ${ }^{35}$ Austoni et a ${ }^{13}$ describe a technique wherein a soft but axially rigid prosthesis is implanted with relaxing tunica albuginea incisions with saphenous vein grafting. In this 145 -patient prospective series, average penile lengthening was reported to be approximately $1.5 \mathrm{~cm}$, and complete satisfaction was expressed in $95 \%$ of patients. Complications of this technique were high, with $5 \%$ of patients experiencing glans paresthesias, $8 \%$ with postoperative infection, and $10 \%$ required repeat circumcision secondary to preputial edema. Mean follow-up was 13 months. ${ }^{13}$ 
Sansalone et $\mathrm{al}^{42}$ have described circumferential tunical incision during penile prosthesis implantation in patients with PD and severe shortening in a multicenter prospective study. Twenty-three patients with PD, refractory ED, and severe penile shortening underwent penile lengthening with circumferential graft and concomitant implantation of an IPP with mean follow-up of 22 months. AMS 700 CX (American Medical Systems, Inc.), Coloplast Titan (Coloplast Corp), and AMS Ambicor two-piece IPP (American Medical Systems, Inc.) devices were utilized. Combined subcoronal and penoscrotal approaches were utilized. An average length gain of $2.8 \mathrm{~cm}$ was recorded and all patients were able to cycle the device and engage in sexual intercourse. Ninety percent of patients were satisfied with the cosmetic and functional result of surgery based on the modified Erectile Dysfunction Index of Treatment Satisfaction (EDITS) questionnaire. Patientreported complications included diminished glans sensitivity in four patients $(20 \%)$ and persistent dorsal curvature of $<15^{\circ}$ in three $(15 \%){ }^{42}$

Those patients presenting with the most complex penile deformities and extensive plaques require plaque incision and grafting at the time of IPP insertion. After the device is implanted, full inflation of the prosthesis and exposure of the area of maximum curvature allows incision over the inflated prosthesis using electrocautery to preserve the integrity of the cylinder. Infrapubic or penoscrotal incisions may be extended to expose the curved area in very proximal curvatures; however, distal curvature requires a countering ventral or circumcoronal incision with penile skin degloving. The deep dorsal vein is ligated and Buck's fascia is dissected away from the dorsal neurovascular bundle to preserve the dorsal penile nerves. Once the tunica albuginea is exposed, the area of maximum curvature is incised with electrocautery until complete straightening has been achieved. If ventral curvature is present, mobilization of the corpus spongiosum and urethra may be required. With the incision completed, the penis will straighten with device inflation. If the defect is large, grafting must be considered. Bertram et a $1^{43}$ suggest that defects that are readily palpable through the replaced skin should be grafted to improve the cosmetic appearance and feel of the prosthesis, while Fishman ${ }^{44}$ suggests that defects $>50 \%$ of the corporeal circumference should be grafted.

Autologous and synthetic graft materials have been used, including dermis and cadaveric pericardium, porcine small intestinal submucosa, and saphenous vein grafts. ${ }^{3,11}$ The grafting technique involves obtaining and tailoring to a size of approximately $25 \%$ larger than the size of the defect to allow for penile extension and contraction. ${ }^{11}$ The graft is then tailored to the size and shape of the defect and secured in place with running sutures. ${ }^{11}$ If the curvature of the distal most portion of the penis persists following the procedure, glanuloplasty performed on the corpora cavernosa lateral to the dorsal neurovascular bundle and ventral corpus spongiosum may improve postoperative results and will eliminate distal penile curvature and supersonic transporter deformity. ${ }^{45}$

Rolle et $\mathrm{al}^{46}$ report success with the so-called "sliding technique", where penile lengthening has been described utilizing a ventro-dorsal incision of the tunica albuginea, penile prosthesis implantation, and double dorsal-ventral patch grafting with porcine small intestinal submucosa. The three patients described in this study had an average length increase of $3.2 \mathrm{~cm}$; all resumed satisfactory sexual intercourse and none had significant loss of sensitivity or any sign of vascular distress of the glans at 13 months follow-up. ${ }^{46}$

Egydio et a ${ }^{17}$ describe a technique to restore penile length by utilizing penile prosthesis placement with concomitant penile lengthening and girth restoration through circular and longitudinal incisions in the tunica albuginea, according to geometric principles with an overall satisfaction rate of approximately $90 \%$. The surgical technique involves calculating the size of the tunical defect preoperatively, so as to save time intraoperatively. In this study of 105 men, the overall satisfaction rate was $89.4 \%$; there was a mean functional penile length gain of $3.6 \mathrm{~cm}$, a patient satisfaction with penile length gain of $95.2 \%$, and $99 \%$ of men were able to perform sexual intercourse with reported satisfaction. ${ }^{47}$

Incision and grafting often requires mobilization of the neurovascular bundle; this procedure has been associated with an increased risk of sensory deficit, and it may require a secondary incision for dorsal access, which is associated with increased morbidity. Because of this increased morbidity, these additional procedures should only be attempted if modeling has failed to correct the curvature and the surgeon is comfortable with penile reconstruction.

\section{Transcorporal techniques}

These minimally invasive techniques attempt to improve the ability of the surgeon to improve the effectiveness of existing techniques or to enhance the ability to excise the plaque without resorting to mobilization of the neurovascular bundle and/or secondary incisions. The "scratch technique" and transcorporal incision have recently been described. ${ }^{14,28}$

\section{Transcorporal incision}

Shaee ${ }^{14}$ describes a transcorporal incision approach to restore straightness and length to the penis prior to calibration 
of the corpora cavernosa, allowing implantation of a longer prosthesis in a straight penis without neurovascular bundle mobilization or a secondary incision. In this 16-patient series, men with dorsal, ventral, and lateral curvatures of varying degrees, as well as some patients with hourglass deformity, underwent transcorporal incision at the point of maximum deformity prior to calibration of the corpora cavernosa. Artificial erection was induced with saline infusion to help identify the point of maximum deformity, as well as to measure the erect shaft length. Via a penoscrotal incision, ventral corporotomies were incised and the corpora were dilated in the usual fashion. Utilizing the concept of "optical corporotomy", plaques were incised at their point of maximum curvature with the assistance of a cystoscope introduced into the corpora cavernosa. Incisions were made across the plaque area utilizing either the cold knife or diathermy electrode. Artificial erection is again induced to ensure correction of the deformity. The corporal length is measured from within, and implantation proceeds in the usual fashion. This technique straightened the penis in all cases. The average follow-up was 14 months, during which time all men resumed sexual activity. No sensory deficits or residual curvature were noted. No patients experienced infection. Pre- and post-transcorporal incision pendulous penile length were measured and compared, with posttranscorporal incision length being $2.1 \mathrm{~cm}(21 \%)$ longer. All patients expressed satisfaction with the outcome; however, no standardized questionnaire was applied to measure patient and partner satisfaction. ${ }^{14}$

\section{The "Scratch" technique}

While modeling alone is able to correct most PD-related curvatures at the time of IPP placement, there exists a nearly $5 \%$ risk of urethral injury. In an attempt to mitigate this risk, Perito et $\mathrm{al}^{28}$ describe a transcorporal plaque disruption to be used as an adjunctive technique to modeling, the so-called "scratch" technique. An artificial erection is created to help identify scar location, and an $80 \mathrm{~mm}$ nasal speculum is passed across the plaque via an infrapubic incision and opened transversely to fracture the plaque along its horizontal axis. A hook-bladed scalpel (\#12 blade) or Metzenbaum scissors are then inserted to internally "scratch", or gently scrape, the plaque along its longitudinal axis. The implant is then placed in standard fashion, and additional modeling may be performed if necessary. The authors assert that the incised plaque will be able to reduce the curvature to $<30^{\circ}$, thereby making plaques more amenable to straightening via modeling, while also decreasing the risk of urethral perforation. ${ }^{23}$

\section{Discussion}

$\mathrm{PD}$ is a heterogeneous disease with variable presentations and patient factors that determine unique surgical management strategies tailored to the needs of each individual patient. The American Urological Association recently published guidelines for the use of prosthesis surgery for patients with PD and ED, or with a deformity sufficient to impair coitus despite pharmacotherapy and/or vacuum device therapy, given the clear benefits of surgery and the low risk of serious adverse events. ${ }^{48}$ Additionally, they noted that clinicians should perform adjunctive intraoperative procedures, such as modeling, plication, or incision/grafting, when significant deformity persists postdevice placement. Each of these recommendations is based on grade $\mathrm{C}$ evidence, with most studies being observational. ${ }^{48}$

We agree with these recommendations and feel that the adjunctive modality of choice for the treatment of persistent deformity post-IPP placement should ultimately be determined by the surgeon's preference and level of comfort with the procedure. Three-piece devices have been shown to be effective with excellent patient satisfaction and functional outcomes, albeit significantly lower than those seen in the non-PD patient, and they have the advantage of accommodating modeling more readily than semirigid prostheses. Several challenges to device placement inherent to the effects of PD on tunical and corporal anatomy likely explain these findings.

Persistent curvature and penile shortening after implantation are the most common reasons for patient dissatisfaction. Several adjunctive procedures have been described to help address persistent deformity and penile shortening, and their outcomes have been validated in several studies. While selecting the adjunctive procedure, surgeon comfort and experience should be paramount. Optimizing functional results and, subsequently, patient satisfaction requires shared decision making between the patient and surgeon in order to manage expectations.

\section{Disclosure}

Culley C Carson III is a consultant for Auxilium and American Medical Systems. Matthew D Lyons and Robert M Coward report no conflicts of interest in this work.

\section{References}

1. Kadioglu A, Akman T, Sanli O, Gurkan L, Cakan M, Celtik M. Surgical treatment of Peyronie's disease: a critical analysis. Eur Urol. 2006;50(2): 235-248.

2. Kumar R, Nehra A. Surgical and minimally invasive treatments for Peyronie's disease. Curr Opin Urol. 2009;19(6):589-594.

3. Carson CC, Levine LA. Outcomes of surgical treatment of Peyronie's disease. BJU Int. 2014;113(5):704-713. 
4. Ralph D, Gonzalez-Cadavid N, Mirone V, et al. The management of Peyronie's disease: evidence-based 2010 guidelines. J Sex Med. 2010; 7(7):2359-2374.

5. Levine LA, Benson J, Hoover C. Inflatable penile prosthesis placement in men with Peyronie's disease and drug-resistant erectile dysfunction: A single-center study. J Sex Med. 2010;7(11):3775-3783.

6. Levine LA, Dimitriou RJ. A surgical algorithm for penile prosthesis placement in men with erectile failure and Peyronie's disease. Int $J$ Impot Res. 2000;12(3):147-151.

7. Scott FB, Bradley WE, Timm GW. Management of erectile impotence. Use of implantable inflatable prosthesis. Urology. 1973;2(1):80-82.

8. Wilson SK, Delk JR. A new treatment for Peyronie's disease: modeling the penis over an inflatable penile prosthesis. J Urol. 1994;152(4): 1121-1123.

9. Wilson SK, Cleves MA, Delk JR 2nd. Ultrex cylinders: problems with uncontrolled lengthening (the S-shaped deformity). J Urol. 1996; 155(1):135-137.

10. Garber BB. Mentor alpha-1 inflatable penile prosthesis cylinder aneurysm: an unusual complication. Int J Impot Res. 1995;7(1):13-16.

11. Carson CC. Penile prosthesis implantation in the treatment of Peyronie's disease and erectile dysfunction. Int J Impot Res. 2000;12 Suppl 4: S122-S126.

12. Kadioglu A, Sanli O, Akman T, Cakan M, Erol B, Mamadov F. Surgical treatment of Peyronie's disease: a single center experience with 145 patients. Eur Urol. 2008;53(2):432-439.

13. Austoni E, Colombo F, Romanò AL, Guarneri A, Kartalas Goumas I, Cazzaniga A. Soft prosthesis implant and relaxing albugineal incision with saphenous grafting for surgical therapy of Peyronie's disease: a 5-year experience and long-term follow-up on 145 operated patients. Eur Urol. 2005;47(2):223-229; discussion 229.

14. Shaeer O. Trans-corporal incision of Peyronie's plaques. J Sex Med 2011;8(2):589-593.

15. Grasso M, Lania C, Fortuna F, Blanco S, Piacentini I. Preservation of cavernosal erectile function after soft penile prosthesis implant in Peyronie's disease: long-term followup. Adv Urol. 2008;2008:646052.

16. Montorsi F, Guazzoni G, Bergamaschi F, Rigatti P. Patient-partner satisfaction with semirigid penile prostheses for Peyronie's disease: a 5-year followup study. J Urol. 1993;150(6):1819-1821.

17. Montorsi F, Guazzoni G, Barbieri L, et al. AMS 700 CX inflatable penile implants for Peyronie's disease: functional results, morbidity and patient-partner satisfaction. Int J Impot Res. 1996;8(2):81-85; discussion 85-86.

18. Akin-Olugbade O, Parker M, Guhring P, Mulhall J. Determinants of patient satisfaction following penile prosthesis surgery. J Sex Med. 2006;3(4):743-748.

19. Chung E, Solomon M, DeYoung L, Brock GB. Comparison between AMS $700^{\mathrm{TM}} \mathrm{CX}$ and Coloplast ${ }^{\mathrm{TM}}$ Titan inflatable penile prosthesis for Peyronie's disease treatment and remodeling: clinical outcomes and patient satisfaction. $J$ Sex Med. 2013;10(11):2855-2860.

20. Morganstern SL. Long-term experience with the AMS 700CX inflatable penile prosthesis in the treatment of Peyronie's disease. Tech Urol. 1997;3(2):86-88.

21. Jena AB, Seabury S, Lakdawalla D, Chandra A. Malpractice risk according to physician specialty. N Engl J Med. 2011;365(7):629-636.

22. Kaplan GW. Malpractice risks for urologists. Urology. 1998;51(2): 183-185.

23. Sunaryo PL, Colaco M, Terlecki R. Penile prostheses and the litigiouspatient: a legal database review. J Sex Med. 2014;11(10): 2589-2594.

24. Sansalone S, Garaffa G, Djinovic R, et al. Simultaneous total corporal reconstruction and implantation of a penile prosthesis in patients with erectile dysfunction and severe fibrosis of the corpora cavernosa. $J$ Sex Med. 2012;9(7):1937-1944.

25. Kadioglu A, Küçükdurmaz F, Sanli O. Current status of the surgical management of Peyronie's disease. Nat Rev Urol. 2011;8(2):95-106.
26. Martínez-Salamanca JI, Mueller A, Moncada I, Carballido J, Mulhall JP. Penile prosthesis surgery in patients with corporal fibrosis: a state of the art review. J Sex Med. 2011;8(7):1880-1889.

27. Vicini P, De Marco F, Antonini G, et al. Use of inflatable penile prostheses AMS CX with momentary squeeze in a patient with Peyronie's disease after removal of two previously implanted penile prostheses. Arch Ital Urol Androl. 2014;86(1):46-47.

28. Perito P, Wilson S. The Peyronie's plaque "scratch": an adjunct to modeling. J Sex Med. 2013;10(5):1194-1197.

29. Segal RL, Burnett AL. Surgical management for Peyronie's disease. World J Mens Health. 2013;31(1):1-11.

30. Yafi FA, Sangkum P, McCaslin IR, Hellstrom WJ. Strategies for penile prosthesis placement in Peyronie's disease and corporal fibrosis. Curr Urol Rep. 2015;16(4):21.

31. Shindel AW, Bullock TL, Brandes S. Urologist practice patterns in the management of Peyronie's disease: a nationwide survey. J Sex Med. 2008;5(4):954-964.

32. Garaffa G, Minervini A, Christopher NA, Minhas S, Ralph DJ. The management of residual curvature after penile prosthesis implantation in men with Peyronie's disease. BJU Int. 2011;108(7):1152-1156.

33. Adibi M, Hudak SJ, Morey AF. Penile plication without degloving enables effective correction of complex Peyronie's deformities. Urology. 2012;79(4):831-835.

34. Hudak SJ, Morey AF, Adibi M, Bagrodia A. Favorable patient reported outcomes after penile plication for wide array of peyronie disease abnormalities. J Urol. 2013;189(3):1019-1024.

35. Kim DH, Lesser TF, Aboseif SR. Subjective patient-reported experiences after surgery for Peyronie's disease: corporeal plication versus plaque incision with vein graft. Urology. 2008;71(4):698-702.

36. Chung PH, Scott JF, Morey AF. High patient satisfaction of inflatable penile prosthesis insertion with synchronous penile plication for erectile dysfunction and Peyronie's disease. J Sex Med. 2014; 11(6):1593-1598

37. Devine CJ Jr, Horton CE. Surgical treatment of Peyronie's disease with a dermal graff. J Urol. 1974;111(1):44-49.

38. Lue TF, El-Sakka AI. Venous patch graft for Peyronie's disease. Part I: technique. J Urol. 1998;160(6 Pt 1):2047-2049.

39. Li CY, Kayes O, Kell PD, Christopher N, Minhas S, Ralph DJ. Penile suspensory ligament division for penile augmentation: indications and results. Eur Urol. 2006;49(4):729-733.

40. Rigaud G, Berger RE. Corrective procedures for penile shortening due to Peyronie's disease. J Urol. 1995;153(2):368-370.

41. Rolle L, Ceruti C, Tamagnone A, et al. T05-O-19 The "Apollo" tissue expander: a novel approach to penile shortening due to fibrosis. Sexologies. 2008;17 Suppl 1:S88.

42. Sansalone S, Garaffa G, Djinovic R, et al. Simultaneous penile lengthening and penile prosthesis implantation in patients with Peyronie's disease, refractory erectile dysfunction, and severe penile shortening. J Sex Med. 2012;9(1):316-321.

43. Bertram RA, Carson CC 3rd, Altaffer LF. Severe penile curvature after implantation of an inflatable penile prosthesis. J Urol. 1988; 139(4):743-745.

44. Fishman IJ. Corporeal reconstruction procedures for complicated penile implants. Urol Clin North Am. 1989;16(1):73-90.

45. Ball TP Jr. Surgical repair of penile "SST" deformity. Urology. 1980;15(6):603-604.

46. Rolle L, Ceruti C, Timpano M, et al. A new, innovative, lengthening surgical procedure for Peyronie's disease by penile prosthesis implantation with double dorsal-ventral patch graft: the "sliding technique" J Sex Med. 2012;9(9):2389-2395.

47. Egydio PH, Kuehhas FE, Sansalone S. Penile length and girth restoration in severe Peyronie's disease using circular and longitudinal grafting. BJU Int. 2013;111(4 Pt B):E213-E219.

48. Nehra A, Alterowitz R, Culkin DJ, et al. Peyronie's disease. J Urol. Epub June 9, 2015. pii: S0022-5347(15)04143-9. 


\section{Publish your work in this journal}

Medical Devices: Evidence and Research is an international, peerreviewed, open access journal that focuses on the evidence, technology, research, and expert opinion supporting the use and application of medical devices in the diagnosis, treatment and management of clinical conditions and physiological processes. The identification of novel

devices and optimal use of existing devices which will lead to improved clinical outcomes and more effective patient management and safety is a key feature. The manuscript management system is completely online and includes a quick and fair peer-review system. Visit http://www. dovepress.com/testimonials.php to read real quotes from authors.

Submit your manuscript here: http://www.dovepress.com/medical-devices-evidence-and-research-journal 